Neurobiology of Disease

Elsevier Editorial System(tm) for

Manuscript Draft

Manuscript Number: NBD-18-594R2

Title: G2019S LRRK2 mutation facilitates $\alpha$-synuclein neuropathology in aged mice

Article Type: Research paper

Keywords: $\alpha$-synuclein; G2019S knock-in mice; iba-1; LRRK2; microglia;

Parkinson's disease

Corresponding Author: Professor Michele Morari,

Corresponding Author's Institution: University of Ferrara

First Author: Salvatore Novello, BSC

Order of Authors: Salvatore Novello, BSc; Ludovico Arcuri, PhD; Sandra Dovero; Nathalie Dutheil; Derya R Shimshek, PhD; Erwan Bezard; Michele Morari

Abstract: Fibrillization of $\alpha$-synuclein is instrumental for the development of Parkinson's disease (PD), thus modulating this process can have profound impact on disease initiation/progression. Here, the impact of the p.G2019S mutation of leucine-rich repeat kinase 2 (LRRK2), which is most frequently associated with familial and sporadic PD, on $\alpha-$ synuclein pathology was investigated. G2019S knock-in mice and wild-type controls were injected with a recombinant adeno-associated viral vector serotype 2/9 (AAV2/9) overexpressing human mutant p.A53T $\alpha$-synuclein (AAV2/9-ho-syn). Control animals were injected with AAV2/9 carrying green fluorescent protein. Motor behavior, transgene expression, $\alpha$-syn and pSer129 a-syn load, number of nigral dopamine neurons and density of striatal dopaminergic terminals were evaluated. To investigate the effect of aging, experiments were performed in 3- and 12-month-old mice, evaluated 20 and 12 weeks after virus injection, respectively. ho-syn overexpression induced progressive motor deficits, loss of nigral dopaminergic neurons and striatal terminals, and appearance of proteinase K-resistant aggregates of pSer129 a-syn in both young and old mice. Although no genotype difference was observed in 3-month-old mice, degeneration of nigral dopaminergic neurons was higher in 12-month-old G2019S knock-in mice compared with age-matched wild-type controls (-55\% vs $-39 \%$, respectively). Consistently, a two-fold higher load of pser129 a-syn aggregates was found in 12-month-old G2019s knock-in mice. We conclude that G2019S LRRK2 facilitates $\alpha$-synucleinopathy and degeneration of nigral dopaminergic neurons, and that aging is a major determinant of this effect. 


\section{DEPARTMENT OF MEDICAL SCIENCES}

\section{Section of Pharmacology \\ UNIVERSITY OF FERRARA}

\section{Michele Morari, PhD}

Associate Professor of Pharmacology

via Fossato di Mortara 19, 44121 Ferrara

ph: +39 0532 455210; fax: +39 0532455205

E-mail:m.morari@unife.it

Professor Tim Greenamyre

Institute for Neurodegenerative Diseases,

University of Pittsburgh,

3501 Fifth Avenue, Suite 7039,

Pittsburgh, PA, USA

Ferrara, 24 August, 2018

Dear Prof Greenamyre,

We would like to thank You, the Editors and Reviewers of Neurobiology of Disease for positive evaluation of the first revision of our manuscript entitled "G2019S LRRK2 mutation facilitates $\alpha$-synuclein neuropathology in aged mice".

We have further modified the Introduction according to the suggestions of Reviewer \#2, essentially quoting our previous finding on pSer1292 levels in G2019 KI mice (Longo et al., 2017) and on LRRK2 co-localization with TH+ in the murine substantia nigra (West et al., 2014).

Changes made have been highlighted in text. A point-to-point reply to Reviewer \#2 has been uploaded in the system.

The Authors declare no competing financial interests.

We now hope that our manuscript will be considered suitable for publication in Neurobiology of Disease and thank you for the attention given to our work.

Sincerely,

Michele Morari

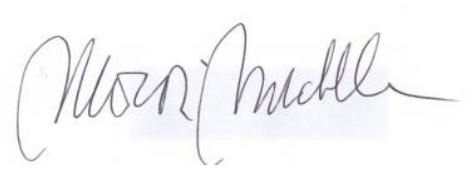




\section{Reply to Reviewers}

Reviewer \#2: The authors should mention, in the introduction, that the Longo 2017 paper showed using immunoblots of striatal lysates that phosphorylation levels of LRRK2 at Ser1292 (pSer1292) are elevated in G2019S knock-in (KI) mice, demonstrating that LRRK2 kinase activity is increased. Unfortunately, it would still be nice to know if LRRK2 kinase activity is increased in the nigra. But, I understand that the lysates may not be available for this study. I also refer the authors to a recent paper that used a proximity ligation assay to assess LRRK2 kinase activity in tissue sections (Di Maio et al. Sci Transl Med 2018). This may be helpful to the authors in the future.

Re: we thank the Reviewer for this valuable information. Indeed, brain lysates are no more available for further analysis of nigral LRRK2 kinase activity. As requested by the Reviewer, we have recalled in Introduction our previous finding that G2019S KI mice have 8-fold greater pSer1292 phosphorylation levels in striatum compared to wild-type controls (Longo et al. 2017), which confirms an elevated LRRK2 kinase activity in G2019S KI mice.

For point 2, the authors did not answer the question- where is LRRK2 expressed in the G2019S knockin mice? If their point is that it should resemble the endogenous expression pattern, at the very least they must cite West et al. 2014 (JCN) that showed in mice, endogenous LRRK2 is expressed in the SNpc, striatum and cortex (figures 1-4). If the authors actually read this paper, they would see that both endogenous expression of LRRK2 in wild type mice was analyzed, as well as overexpressed LRRK2 in BAC transgenic mice.

Re: As requested by the Reviewer, we have added to Introduction a sentence quoting previous work of West and collaborators (West et al., JCN 2014) showing expression of endogenous LRRK2 also in dopaminergic neurons of substantia nigra. 


\section{Highlights}

AAV2/9-h $\alpha$-syn injection causes dopamine neuron loss and $\alpha$-syn aggregates appearance Aged G2019S LRRK2 KI mice are more prone to develop $\alpha$-syn toxicity than WT mice Larger amount of $\alpha$-syn aggregates are present in aged G2019S LRRK2 KI mice No microglial response is observed 3-5 months after AAV2/9-h $\alpha$-syn injection 


\title{
G2019S LRRK2 mutation facilitates $\alpha$-synuclein neuropathology in aged mice
}

Salvatore Novello ${ }^{1}$, Ludovico Arcuri ${ }^{1}$, Sandra Dovero ${ }^{2,3}$, Nathalie Dutheil ${ }^{2,3}$, Derya R. Shimshek ${ }^{4}$, Erwan Bezard $^{2,3}$, and Michele Morari ${ }^{1}$

${ }^{1}$ Department of Medical Sciences, Section of Pharmacology, University of Ferrara and National Institute of Neuroscience, Ferrara, Italy.

${ }^{2}$ Université de Bordeaux, Institut des Maladies Neurodégénératives, UMR 5293, F-33000 Bordeaux, France.

${ }^{3}$ CNRS, Institut des Maladies Neurodégénératives, UMR 5293, F-33000 Bordeaux, France.

${ }^{4}$ Department of Neuroscience, Novartis Institutes for BioMedical Research, Novartis Pharma AG, 4002 Basel, Switzerland

\author{
Correspondence author \\ Michele Morari \\ Department of Medical Sciences, Section of Pharmacology \\ University of Ferrara, via Fossato di Mortara 17-19, 44121 Ferrara (Italy) \\ Phone: +39-0532-455210 \\ Fax: +39-0532-455205 \\ E-mail:m.morari@unife.it
}

\begin{abstract}
Abbreviations: AAV2/9, adeno-associated viral vector pseudotype 2/9; DA, dopamine; G2019S knock-in, G2019S KI; h $\alpha$-syn, p.A53T human $\alpha$-synuclein; ionized calcium binding adaptor molecule 1, iba-1; LRRK2, leucine-rich repeat kinase 2; Ser129 $\alpha$-syn, $\alpha$-synuclein phosphorylated at Serine 129; PD, Parkinson's disease; SNc, substantia nigra compacta; TH, tyrosine hydroxylase.
\end{abstract}

Declaration of interests: none 


\begin{abstract}
Fibrillization of $\alpha$-synuclein is instrumental for the development of Parkinson's disease (PD), thus modulating this process can have profound impact on disease initiation/progression. Here, the impact of the p.G2019S mutation of leucine-rich repeat kinase 2 (LRRK2), which is most frequently associated with familial and sporadic PD, on $\alpha$-synuclein pathology was investigated. G2019S knock-in mice and wild-type controls were injected with a recombinant adeno-associated viral vector serotype 2/9 (AAV2/9) overexpressing human mutant p.A53T $\alpha$-synuclein (AAV2/9ha-syn). Control animals were injected with AAV2/9 carrying green fluorescent protein. Motor behavior, transgene expression, $\alpha$-syn and pSer129 $\alpha$-syn load, number of nigral dopamine neurons and density of striatal dopaminergic terminals were evaluated. To investigate the effect of aging, experiments were performed in 3- and 12-month-old mice, evaluated 20 and 12 weeks after virus injection, respectively. ha-syn overexpression induced progressive motor deficits, loss of nigral dopaminergic neurons and striatal terminals, and appearance of proteinase K-resistant aggregates of pSer129 $\alpha$-syn in both young and old mice. Although no genotype difference was observed in 3month-old mice, degeneration of nigral dopaminergic neurons was higher in 12-month-old G2019S knock-in mice compared with age-matched wild-type controls (-55\% vs $-39 \%$, respectively). Consistently, a two-fold higher load of pSer129 $\alpha$-syn aggregates was found in 12-month-old G2019S knock-in mice. We conclude that G2019S LRRK2 facilitates $\alpha$-synucleinopathy and degeneration of nigral dopaminergic neurons, and that aging is a major determinant of this effect.
\end{abstract}

Keywords: $\alpha$-synuclein, G2019S knock-in mice, iba-1, LRRK2, microglia, Parkinson's disease. 


\section{Introduction}

Parkinson's disease (PD) is a progressive neurodegenerative disorder neuropathologically characterized by the loss of dopamine (DA) neurons in the substantia nigra pars compacta (SNc) and the appearance of proteinaceous intracytoplasmic aggregates named Lewy bodies (LB) in surviving neurons (Poewe et al., 2017). The main component of LB is $\alpha$-synuclein ( $\alpha$-syn) (Spillantini et al., 1997), a presynaptic protein with high propensity to form fibrillary structures and aggregates (Bengoa-Vergniory et al., 2017; Lashuel et al., 2013).

$\alpha$-syn fibrillization is a key process in PD since it can both initiate and sustain the pathology (Lashuel et al., 2013). In fact, mutations (Polymeropoulos et al., 1997) or duplications/triplications (Singleton et al., 2003) of the SNCA gene coding for $\alpha$-syn are causative of familial PD, and mutations in regulatory regions upstream of the SNCA gene are risk variants for idiopathic PD (Nalls et al., 2014). Moreover, misfolded $\alpha$-syn can spread from one neuron to another through a process of exocytosis/endocytosis, acting as a seed for endogenous $\alpha$-syn fibrillization (Angot et al., 2010; Brundin et al., 2010). Thus, any factor influencing $\alpha$-syn fibrillization may have a profound impact on disease onset and progression (Brundin et al., 2017). In this context, the role of leucinerich repeat kinase 2 (LRRK2) has been investigated (Greggio et al., 2011; Guerreiro et al., 2013). LRRK2 is a 2527 amino acid long, multidomain protein encompassing a kinase and a GTPase domain surrounded by protein-protein interaction domains (Cookson, 2010; Mata et al., 2006). Mutations in the lrrk2 gene are associated with familial, autosomal dominant forms of PD (PaisanRuiz et al., 2004; Zimprich et al., 2004) and are considered a risk factor for idiopathic PD (Satake et al., 2009). The most frequent mutation (Correia Guedes et al., 2010) is the c.6055G>A, which results in a substitution of a glycine with a serine in position 2019 (p.G2019S), situated in the kinase domain. This mutation confers the protein a higher kinase activity and in vitro neurotoxicity (Greggio et al., 2006; West et al., 2005; Yao et al., 2010), thus representing an ideal target for therapeutic intervention. G2019S LRRK2-associated PD is clinically and neuropathologically 
similar to idiopathic PD, being characterized by nigral DA neuron degeneration and the presence of LBs (in the vast majority of cases) (Kalia et al., 2015; Marras et al., 2011).

Previous genetic studies have provided inconsistent evidence that LRRK2 impacts $\alpha$-syn neuropathology. In particular, double transgenic (TG) mice overexpressing human A53T $\alpha$-syn in combination with human LRRK2 wildtype (WT) or LRRK2 G2019S presented exacerbated $\alpha$-syn pathology and neurotoxicity in striatal and cortical neurons (Lin et al., 2009). Another study on similar strains of double mutant mice failed to show that G2019S LRRK2 enhances the nigrostriatal degeneration and the accompanying $\alpha$-syn pathology induced by overexpression of human A53T $\alpha$ syn (Daher et al., 2012). Among the few brainstem areas investigated, a modest increase in $\alpha$-syn aggregates was observed in the reticular formation but not in the lateral vestibular or lateral cerebellar nuclei (Daher et al., 2012). Similar negative results were reported analyzing the cortex, brainstem and spinal cord of double A53T/WT LRRK2 or A53T/G2019S LRRK2 TG mice (Herzig et al., 2012). Instead, a significant interplay between LRRK2 and $\alpha$-syn was reported using a different model of synucleinopathy (Daher et al., 2015). BAC rats overexpressing human G2019S LRRK2, injected with an adeno-associated 2/1 virus (rAAV2/1) carrying human WT $\alpha$-syn (Daher et al., 2014) showed a larger loss of nigral DA neurons and associated microgliosis compared to non TG rats injected with the same construct (Daher et al., 2015).

These genetic and pharmacological studies, despite suggestive of a G2019S effect on $\alpha$-syn pathology, were performed in animals where the transgenes were not always co-expressed in brain areas relevant to PD. In fact, significant levels of the transgene were detected in the SNc of BAC G2019S overexpressing rats (Daher et al., 2015) whereas G2019S overexpressing mice showed null (Daher et al., 2012; Herzig et al., 2012) or very limited (<5\%) (Lin et al., 2009) nigral expression of human G2019S. We therefore sought to investigate the interplay between G2019S LRRK2 and $\alpha$ syn in G2019S knock-in (KI) mice. These mice express only the G2019S mutant form, and at physiological levels, in a pattern resembling that of endogenous LRRK2, characterized by 
significant levels also in tyrosine hydroxylase (TH) positive neurons of substantia nigra (West et al., 2014). Moreover, using immunoblots of striatal lysates, we previously reported that these mice have 8-fold greater pSer1292 LRRK2 phosphorylation levels compared to wild-type controls (Longo et al., 2017), confirming an elevated LRRK2 kinase activity in G2019S KI mice. Thus, we injected recombinant adeno-associated viral (AAV) vector serotype 2/9 carrying human p.A53T $\alpha$-syn (AAV2/9 h $\alpha$-syn) or GFP (green fluorescent protein) as a control (Bourdenx et al., 2015) in the SNc of 3 month-old G2019S KI and age-matched wild-type mice. This procedure leads to overexpression of ha-syn in dopamine neurons (Bourdenx et al., 2015). Moreover, since we previously reported that G2019S KI mice develop synaptic dysfunction and $\alpha$-syn inclusions over aging (Longo et al., 2017), we repeated the AAV injection procedures in a cohort of 12-month-old mice, to investigate the effect of aging over the G2019S LRRK2/ $\alpha$-syn interplay. Motor activity, nigrostriatal degeneration and synucleinopathy were evaluated at 20 weeks (3-month cohort) or 12 weeks (12-month cohort) after virus injection. Since microglia can contribute to dopaminergic neurodegeneration (Kim et al., 2018), in both cohorts of animals a preliminary analysis of the microglia morphology was also performed.

\section{Materials and methods}

\section{Subjects}

Experimental procedures involving the use of animals complied with the ARRIVE guidelines and the EU Directive 2010/63/EU for animal experiments and were approved by the Ethical Committee of the University of Ferrara and the Italian Ministry of Health (license 714/2017-PR). Male homozygous LRRK2 G2019S KI mice, backcrossed on a C57BL/6J background, were used. Founders were obtained from Novartis Institutes for BioMedical Research, Novartis Pharma AG (Basel, Switzerland) (Herzig et al., 2011), and colonies established at the vivarium of the University of Ferrara. A colony of non-TG WT mice was obtained from heterozygous breeding. Control male 
WT mice were then obtained from homozygous breeding. Mice were kept under regular lighting conditions (12 h light/dark cycle) and given food and water ad libitum. Adequate measures were taken to minimize animal pain and discomfort.

\section{Behavioral test}

Drag test. This test (modification of the "wheelbarrow" test) (Schallert et al., 1979), measures the ability of the mouse to balance its body posture using forelimbs in response to an externally imposed dynamic stimulus (backward dragging) (Marti et al., 2005; Marti et al., 2004; Viaro et al., 2008). Each mouse was gently lifted from the tail (leaving the forepaws on the table) and dragged backwards at a constant speed (about $20 \mathrm{~cm} / \mathrm{sec}$ ) for a fixed distance $(100 \mathrm{~cm})$. The number of touches made by each forepaw was counted by two separate observers. Since values did not significantly differ between the left and right forepaw, data were pooled together.

Rotarod test. This test analyzes the ability of the mouse to run on a rotating cylinder (diameter 8 $\mathrm{cm}$ ) and provides information on different motor parameters such as coordination, gait, balance, muscle tone and motivation to run (Rozas and Labandeira Garcia, 1997). The fixed-speed rotarod test was employed according to a previously described protocol (Marti et al., 2004; Viaro et al., 2008). Mice were tested in a stepwise mode at increasing speeds (usually from 5 to $45 \mathrm{rpm}$; $180 \mathrm{sec}$ each), and time spent on the rod calculated (in sec).

\section{AAV2/9-ho-syn vector production and injection}

Recombinant AAV2/9-h $\alpha$-syn vectors driven by the synapsin-I promoter were produced and purified as already described (Bourdenx et al., 2015; Engeln et al., 2013; Zolotukhin et al., 1999). Briefly, vectors were transfected into HEK-293 T/17 cells (ATCC, Teddington, UK) for three times using a polyethylenimine solution. Seventy-two hours after transfection, cells were re-suspended in lysis buffer (150 mM NaCl, $50 \mathrm{mM}$ Tris- $\mathrm{HCl} \mathrm{pH} 8.5)$, and then lysed using a freeze-thaw cycle ($\left.80^{\circ} \mathrm{C} / 37^{\circ} \mathrm{C}\right)$. The obtained supernatant was purified by iodixanol gradient step centrifugation, and finally aliquoted and kept in stock at $-80^{\circ} \mathrm{C}$. 
Nine 3-month-old and ten 12-month-old G2019S KI mice, and equal numbers of age-matched WT mice, received a bilateral SNc stereotaxic injection of AAV2/9-h $\alpha$-syn $\left(2.35 \times 10^{13}\right.$ genome containing particles/ $\mu \mathrm{l} ; 1 \mu \mathrm{l}$ ), under isoflurane anesthesia (Arcuri et al., 2016). As a control, nine 3month-old and ten 12-month-old G2019S KI mice, and equal numbers of age-matched WT mice, received a bilateral SNc stereotaxic injection of AAV-GFP. Virus was injected with a glass syringe at a flow rate of $0.5 \mu 1 / \mathrm{min}$ and was left in place for additional $4 \mathrm{~min}$ to prevent backflush. Coordinates from bregma were (in $\mathrm{mm}$ ): antero-posterior -3.3 ; medio-lateral \pm 1.25 ; dorso-ventral 4.6.

\section{Immunohistochemistry}

Mice were deeply anesthetized with isoflurane and transcardially perfused with Phosphate Buffer Solution (PBS) and then with $4 \%$ paraformaldehyde in PBS (0.1 M, pH 7.4). Brains were removed, transferred to a $30 \%$ sucrose solution in PBS for cryoprotection and then stored at $-80^{\circ} \mathrm{C}$.

TH, iba-1, $\alpha$-syn and pSer129 $\alpha$-syn immunohistochemistry. Fifty micrometer free-floating sections of striatum (AP from +1.0 to -1.25 from bregma) and SNc (AP from -3.16 to -3.52 from bregma (Paxinos and Franklin, 2001) were rinsed in PBS and incubated for $30 \mathrm{~min}$ at room temperature with a blocking solution (PBS+BSA 1:50+Triton X100 0.3\%) and then incubated with a rabbit polyclonal antibody raised against TH (ab112; 1:750 in BSA 1\% PBST; Abcam, Cambridge, UK), total $\alpha$-syn (ab52168; 1:200 in BSA 1\% PBST; Abcam, Cambridge, UK), pSer129 $\alpha$-syn (ab51253; 1:250 in BSA 1\% PBST; Abcam, Cambridge, UK), human $\alpha$-syn (ho-syn) (ab138501; 1:150 in BSA 1\% PBST; Abcam, Cambridge, UK) or ionized calcium binding adaptor molecule 1 (iba-1) (ab178846; 1:2000 in BSA 1\% PBST; Abcam, Cambridge, UK) overnight at room temperature. Sections were then rinsed and incubated for 1 hour with an anti-rabbit HRP-conjugated secondary antibody (ab6721, 1:500 in BSA 1\% PBST; Abcam, Cambridge UK) and revealed by a DAB substrate kit (ab64238, Abcam, Cambridge, UK). Sections were mounted on gelatinized slides, dehydrated and coverslipped for further analysis. 
To quantify the levels of expression of $\alpha$-syn, pSer129 $\alpha$-syn, h $\alpha$-syn, the semi-stereological method described by Bourdenx et al. (Bourdenx et al., 2015) was employed. This method has been rigorously standardized; in fact, all serial striatal slices for each animal were taken, marked, put in the same well and exposed to $\mathrm{DAB}$ for 1 minute (according to the data sheet of Abcam DAB substrate Kit). After being mounted, slides were scanned and the representative surface of the staining in each $\mathrm{SN}$ section was determined using a color threshold, then the area was sampled (probes of $50 \times 40 \mu \mathrm{m}$, space $150 \times 120 \mu \mathrm{m}$ ). The Cavalieri principle was applied to evaluate the representative volume of $\alpha$-syn or pSer129 $\alpha$-syn expression for each SN. The SN volume obtained from the $\alpha$-syn staining was used to calculate the pSer129 $\alpha$-syn expression as a percentage, thus allowing the comparison between groups.

Treatment with proteinase $K(P K)$. Tissue sections were incubated for 5 minutes with PK (ab64220; Abcam, Cambridge, UK) at room temperature. At the end of the incubation, sections were rinsed in PBS and processed as described above using rabbit polyclonal antibody for pSer129 $\alpha$-syn (ab51253; 1:200 in BSA 1\% PBST; Abcam, Cambridge, UK).

\section{Stereology and neuron counting}

Stereological analysis was performed counting $\mathrm{TH}+$ neurons (phenotypic marker) and cresyl violet stained cells (structural marker) in SNc, according to an unbiased stereological sampling method based on optical fractionator stereological probe (Larsen et al., 1998). A Leica DM6B motorized microscope (Leica Microsystems, Milan, Italy) coupled with a Stereo Investigator software (MBF Europe, Delft, The Netherlands) was used. Counting was performed on at least 5 consecutives 50 $\mu \mathrm{m}$ thick slices, magnified at $63 \mathrm{X}$.

\section{TH quantification in striatum}

Images were taken at $2.5 \mathrm{X}$ magnification with a Leica DM6B motorized microscope, and optical densitometry analyzed off-line as grey level with ImageJ using the corpus callosum as background.

\section{Quantification of ho-syn, total $\alpha$-syn and pSer129 $\alpha$-syn expression}


Images were obtained using a Leica DM6B motorized microscope, and later analyzed with ImageJ software. SNc and striatum limits were delineated to obtain a representative surface of ha-syn, total $\alpha$-syn and pSer129 $\alpha$-syn expression. Finally, the immunopositive surface as percentage of total $\mathrm{SNc}$ area was calculated, in order to compare the different treatments.

\section{Iba-1 analysis}

Three consecutives sections were mounted on a coverslip and images were obtained using a Leica DM6B motorized microscope, and later analyzed with ImageJ software. Iba- $1+$ cells were binarized and number of cells and area of surface occupied in pixels ${ }^{2}$ were quantified in order to compare the different treatments.

\section{Data presentation and statistical analysis}

Motor performance in the drag and rotarod test was presented as absolute values (calculated as number of steps or time on rod in sec) and analysed by two-way repeated measure (RM) ANOVA followed by the Bonferroni test. All the other data were analysed by one-way ANOVA followed by the Bonferroni test. Data obtained from stereological counting were expressed as absolute values (number of cells), density of striatal TH terminals was expressed as absolute data (mean absolute value of grey scale between the two striata), density of $\alpha$-syn aggregates was expressed as area of threshold, data from iba- $1+$ cells were expressed as absolute value and as area occupied in pixel $^{2}$. P values $<0.05$ were considered to be statistically significant.

\section{Results}

Injection of AAV2/9-ho-syn in 3-month-old mice

G2019S KI mice were injected with AAV2/9-h $\alpha$-syn to verify whether the increase of LRRK2 kinase activity accelerates h $\alpha$-syn-induced neuropathology. Mice injected with h $\alpha$-syn displayed progressive motor deficits in the drag test whereas mice injected with GFP showed relatively stable performance over the 20-week observation period (treatment effect $\mathrm{F}_{3,5}=20.04, \mathrm{p}<0.0001$; time 
effect $\mathrm{F}_{5,145}=21.23, \mathrm{p}<0.0001$; time $\mathrm{x}$ treatment interaction $\mathrm{F}_{15,145}=2.38, \mathrm{p}=0.0005$ ). Stepping was significantly reduced starting from 8 weeks after virus injection with stable levels attained after 12 weeks onwards (-40\% from baseline) (Fig 1A). The time-courses of stepping activity of G2019S KI and WT mice injected with AAV2/9-h $\alpha$-syn were superimposable, indicating no genotype susceptibility. Stereological analysis revealed a similar number of DA neurons in SNc of GFPinjected G29019S KI and WT mice (4883 \pm 176 vs $5112 \pm 320$; Fig 2A-E). AAV2/9-h $\alpha$-syn injected G29019S KI and WT mice had a similar 50\% loss of DA neurons in SNc (Fig. 1B). Consistently, optical density of TH+ striatal nerve terminals revealed that AAV2/9-h $\alpha$-syn injected G29019S KI and WT mice had 60-70\% loss of striatal TH signal, again without difference between genotypes (Fig. 1C).

The efficiency of AAV2/9 injections was evaluated by the expression patterns of ha-syn (Fig. 2). As expected, only mice injected with AAV2/9-h $\alpha$-syn showed a significant ho-syn labelling in SNc. Strong labelling was also detected in striatum (Fig. 3F-I), indicating the diffusion of ha-syn from SNc to anatomically connected areas. No difference in ha-syn load between genotypes was found in SNc or striatum (Fig. 2A, B).

To confirm the occurrence of $\alpha$-syn pathology, the levels of pSer129 $\alpha$-syn, i.e. the most prominent form of $\alpha$-syn present in LB (Anderson et al., 2006; Fujiwara et al., 2002), were measured. As shown in Fig 3, total $\alpha$-syn labelling, which encompasses both human and endogenous $\alpha$-syn due to the lack of antibody selectivity, was 2.5 -fold greater in the SNc (Fig. 3A) and 1.5-fold greater in the striatum (Fig 3B) of AAV2/9-h $\alpha$-syn injected mice compared to control mice, irrespective of genotype. pSer129 $\alpha$-syn labelling was also 1.5-fold higher in the SNc (Fig. 3C) and 4-fold higher in the striatum (Fig. 3D) of AAV2/9-h $\alpha$-syn injected mice compared to AAV GFP mice, again irrespective of genotype.

To ascertain whether pSer129 $\alpha$-syn labelling was constituted by soluble, monomeric or insoluble, oligomeric $\alpha$-syn forms, slices were treated with proteinase K (Fig. 4). After PK treatment, a 
significant pSer129 $\alpha$-syn signal was detected in both the SNc (Fig. 4A) and striatum (Fig. 4B) of AAV2/9-h $\alpha$-syn injected mice indicating the presence of $\alpha$-syn aggregates. However, the amount was similar in G2019S KI and WT mice, indicating that the mutation did not facilitate $\alpha$-syn aggregation in 3-month-old mice.

Finally, it was investigated whether $\alpha$-syn pathology was accompanied by a microglial response (Supplementary Fig. 1). AAV2/9-ha-syn injection did not change the number and area occupied by iba-1+ cells in SNc (Supplementary Fig 1A-B) or striatum (Supplementary Fig 1C-D), indicating the absence of sustained microgliosis.

\section{Injection of AAV2/9-ho-syn in 12-month-old mice}

Data obtained in 3-month-old mice indicate that G2019S does not accelerate $\alpha$-syn toxicity. We then sought to investigate whether G2019S could play a significant contribution in older animals. As aging is a major risk factor in PD and, in addition, we observed synaptic dysfunction and a pSer129 $\alpha$-syn overload in the striatum of 12-month-old G2019S KI mice, we used older mice to investigate the contribution of age on pathology in our AAV model.

Analysis of motor behaviour showed that 12-month-old AAV2/9-ho-syn injected mice displayed motor deficits in the drag test (treatment $\mathrm{F}_{3,3}=24.24, \mathrm{p}<0.0001$, time $\mathrm{F}_{3,93}=18.31, \mathrm{p}<0.0001$, time $\mathrm{x}$ treatment interaction $\mathrm{F}_{9,93}=4.55, \mathrm{p}<0.0001$; Fig 5A). Stepping was similarly impaired in both genotypes (20-30\%), a significant effect being observed already at 4 weeks after injection (Fig. 5A). The same pattern was confirmed by analysis of global motor activity in the rotarod test (treatment $\mathrm{F}_{3,3}=8.41, \mathrm{p}=0.0003$, time $\mathrm{F}_{3,93}=3.81, \mathrm{p}=0.0126$, time $\mathrm{x}$ treatment interaction $\mathrm{F}_{9,93}=3.80, \mathrm{p}=0.0004$; Fig. 5B).

Stereological analysis performed 12 weeks after surgery showed that 12-month-old GFP-injected G2019S KI and WT mice had a similar number of nigral DA neurons $(4291 \pm 167$ and $4429 \pm 242$, respectively; Fig. 5C). However, G2019S KI mice showed significantly larger $\left(\mathrm{F}_{3,31}=44.16\right.$, $\mathrm{p}<0.0001)$ nigral degeneration compared to WT mice $(-55 \%$ vs $-39 \%, 1944 \pm 168$ vs $2729 \pm 177$ DA 
cells, respectively; Fig. 5C). Nonetheless, striatal TH density analysis revealed that both groups of animals displayed a significant reduction (28\%) of TH+ terminals (Fig. 5D).

Similar to 3-month-old mice, AAV2/9-h $\alpha$-syn injection caused comparable expression of ha-syn (Fig. 6) in SNc (Fig. 6A) and striatum (Fig. 6B) of G29019S KI and WT mice, suggesting comparable transduction efficiencies. Likewise, as in the 3-month-old cohort, total $\alpha$-syn load (Fig. 7) was $~ 2$-fold elevated in the SNc (Fig. 7A) and striatum (Fig. 7B) of AAV2/9-ho-syn injected mice compared to GFP-injected mice. However, different from 3-month-old mice, significant changes in the levels of pSer129 $\alpha$-syn in SNc (treatment $F_{3,32}=1393 p<0.0001$ ) and striatum (treatment $\mathrm{F}_{3,32}=524.8, \mathrm{p}<0.0001$ ) were found. In particular, in line with our previous study (Longo et al., 2017), GFP-treated G2019S KI mice had 3-fold higher levels of pSer129 $\alpha$-syn in SNc (Fig. 7C) and striatum (Fig. 7D) compared to GFP-treated WT mice. Moreover, G2019S KI mice injected with AAV2/9-h $\alpha$-syn showed a 2-fold larger area of signal in both areas with respect to WT mice injected with the same construct.

The area occupied by PK-resistant pSer129 $\alpha$-syn aggregates was then analysed (Fig. 8). Interestingly, upon PK treatment no difference in pSer129 $\alpha$-syn load between G2019S KI and WT could be observed anymore for both brain areas, both in SNc (Fig 8A-B) and striatum (Fig. 8C-D). However, there was still a doubling of pSer129 $\alpha$-syn levels detectable in G2019S KI versus WT mice injected with AAV2/9-ho-syn.

Finally, we investigated the microglial response in this cohort of 12-month-old mice (Supplementary Fig. 2). No changes in the number and area occupied by Iba-1+ microglial cells in SNc (Supplementary Fig 2A-B) or striatum (Supplementary Fig 2C-D), were detected between AAV2/9-ho-syn injected and AAV GFP injected mice, again suggesting no active microglial response at 12 weeks after viral injection. 


\section{Discussion}

Major finding of the present study is that G2019S KI mice are more susceptible than WT controls to develop nigral DA neuron degeneration and synucleinopathy after injection of ha-syn delivered via AAV2/9 vector. The G2019S effect emerges at 12-months of age, further strengthening the role of aging in G2019S-induced synucleinopathy (Longo et al., 2017).

Viral vectors are an efficient way to allow overexpression of native or mutant $\alpha$-syn in nigral DA neurons of rats, mice and nonhuman primates (for reviews see (Low and Aebischer, 2012; Ulusoy et al., 2010; Van der Perren et al., 2015). Consistent with previous studies (Bourdenx et al., 2015; Ip et al., 2017; Lauwers et al., 2003; Song et al., 2015; Svarcbahs et al., 2016; Thome et al., 2015), AAV2/9-ha-syn injection resulted in efficient expression of the human transgene in SN and, after diffusion, in striatum. Accordingly, degeneration of nigral DA neurons (Bourdenx et al., 2015; Ip et al., 2017; Lauwers et al., 2003; Song et al., 2015; Svarcbahs et al., 2016; Thome et al., 2015) and $\alpha$-syn pathology (increased pSer129 $\alpha$-syn levels and PK-resistant $\alpha$-syn aggregates) appeared. Different from studies where unilateral injection was performed (Bourdenx et al., 2015; Ip et al., 2017; Lauwers et al., 2003; Svarcbahs et al., 2016; Thome et al., 2015), the bilateral AAV2/9 injection caused a larger reduction of nigral DA neurons (50\% vs 20-30\%) (also see (Song et al., 2015). Consistent with such higher levels of neurodegeneration, deficits in stepping (drag test) and global motor (rotarod test) activity were observed both in 3month-old and 12-month-old mice, with the difference that in older mice motor deficits appeared earlier, i.e. already at 4 weeks after intranigral AAV2/9 injection, perhaps suggesting an acceleration of the neurotoxic process associated with aging.

The finding that 12-month-old G2019S KI mice were more prone to $\alpha$-syn toxicity cannot be attributed to different efficiencies of transgene expression (or other surgery variables) since hasyn expression and diffusion were similar among the different cohorts of mice. Thus, the neuropathological changes between 12-month-old G2019S KI and WT mice are likely due to 
LRRK2 mutation. This corroborates previous reports that WT LRRK2/A53T $\alpha$-syn and G2019S LRRK2/A53T $\alpha$-syn double TG mice (Lin et al., 2009) or BAC G2019S KI rats injected with a AAV2 $\alpha$-syn (Daher et al., 2015) display greater loss of nigral DA neurons compared to controls. Greater nigral degeneration in 12-month-old G2019S KI mice was associated with greater load of PK-resistant, insoluble pSer129 $\alpha$-syn aggregates. This is in line with the finding that $\alpha$-syn fibrils injection in BAC G2019S overexpressors favours pSer129 $\alpha$-syn inclusions in DA neurons (Volpicelli-Daley et al., 2016), although in this model of synucleinopathy no overt nigro-striatal degeneration was observed (at 4 weeks after injection). Therefore, since the AAV h $\alpha$-syn model is a model for $\alpha$-syn aggregation but not fibrillization, our study would suggest that in aged G2019S KI mice, excessive deposition of pSer129 $\alpha$-syn aggregates in SNc accelerates DA neuron loss. In fact, expression of G2019S LRRK2 in C. elegans caused agedependent accumulation of $\alpha$-syn and degeneration of DA neurons (Saha et al., 2015). $\alpha$-syn aggregates per se can disrupt cellular homeostasis, altering cellular trafficking (Giasson and Lee, 2003; Stefanis, 2012). Among the possible mechanisms through which G2019S LRRK2 accelerates $\alpha$-syn synucleinopathy, we investigated microglial activation. In fact, it is established that LRRK2 promotes microglial activation and the release of pro-inflammatory cytokines (Gillardon et al., 2012; Moehle et al., 2012; Russo et al., 2015) and that G2019S mutation amplifies this response (Kim et al., 2012; Moehle et al., 2015). Another mechanisms has been recently proposed by a study showing that G2019S LRRK2 increases phagocytic activity of microglia and macrophages through stabilization of WAVE2 complex, which is directly linked to DA cell death (Kim et al., 2018). Consistent with those reports, microgliosis was associated with the greater loss of DA neurons observed in G2019S LRRK2/A53T $\alpha$-syn double TG mice (Lin et al., 2009) or BAC G2019S rats injected with AAV $\alpha$-syn (Daher et al., 2015). Different from these reports, however, we failed to detect an increased number of Iba-1+ cells in both the SN and striatum of WT and G2019S KI mice injected with AAV-ho-syn, regardless of the age 
examined. Lack of microglial activation (Litteljohn et al., 2018) or even inhibition of microglial motility (Choi et al., 2015) have been reported in G2019S LRRK2 models. However, we should consider that in the AAV model of synucleinopathy, the number of microglia cells appears to be transiently elevated only at 4-8 weeks after injection (Chung et al., 2009; Daher et al., 2015; Sanchez-Guajardo et al., 2010), consistent with a role of microglia in early stages of AAV $\alpha$-syn toxicity. Since we performed the microglia analysis at 12 or 20 weeks after AAV injection, we might have missed the initial microglial response. We should also consider that in previous studies a sustained microglial activation was observed in the presence of elevated levels of G2019S LRRK2 (either genetically- or virus-induced) which might have further amplified such response.

The lack of a sustained microglial response and the age-dependence of the G2019S KI pattern of response would suggest other mechanisms underlying the G2019S effect. In particular, G2019S LRRK2 can potently regulate cellular proteostasis through autophagy pathways. In fact, $\alpha$-syn is degraded mainly via chaperone-mediated autophagy (Cuervo et al., 2004; Lee et al., 2004) and G2019S LRRK2 impairs $\alpha$-syn clearance (Orenstein et al., 2013; Saha et al., 2015) by promoting $\alpha$-syn oligomerization at the lysosomal membrane (Orenstein et al., 2013). Indeed, G2019S LRRK2 has been shown to impair lysosomal $\alpha$-syn degradation and cause endogenous $\alpha$-syn accumulation, an effect reversed by LRRK2 kinase inhibitors, in primary cortical neurons (Schapansky et al., 2018). Interestingly, aging is accompanied by impairment of chaperonemediated autophagy and proteasome clearance (Mattson and Magnus, 2006; Xilouri and Stefanis, 2016), which leads to the accumulation of $\alpha$-syn aggregates, at least in primates (Chu and Kordower, 2007). Thus, the increased deposition of $\alpha$-syn aggregates in aged G2019S KI mice might be due to a detrimental effect of G2019S LRRK2 combined with the reduced brain capability to handle $\alpha$-syn overload of the aged brain. The fact that we did not observe a genotype effect in young G2019S KI mice, might thus depend on a greater capability of young 
mice to cope with changes of $\alpha$-syn overload. In support of the role of aging in G2019S LRRK2 pathology, a recent study reported nigral DA loss and prolonged microgliosis in 19-month-old but not 2-3-month-old WT mice intrastriatally-injected with an adenoviral vector carrying human G2019S LRRK2 (Kritzinger et al., 2018).

G2019S KI mice, therefore, might represent a model recapitulating the effect of aging (the major risk factor in PD) over the interaction between a pathogenic genetic component (the G2019S mutation) and a PD-relevant trigger (h $\alpha$-syn). Nonetheless, there seems to be a dissociation between neurodegeneration and synucleinopathy in striatum where, different from SNc, no greater loss of DA terminals was detected in G2019S KI mice compared to controls. It is possible that, consistent with the anterograde nature of the AAV2/9-h $\alpha$-syn toxicity process, the greater loss of nigral DA cell bodies in G2019S KI mice needs more time to translate into a significant change at the striatal terminal level. Alternatively, we could speculate that the toxic effect exerted by G2019S LRRK2 at the terminal levels is compensated.

\section{Concluding remarks}

Evidence was presented that G2019S KI mice are more susceptible to the toxic effects of ha-syn delivered via AAV2/9, that this effect is age-dependent and associated with greater load of insoluble pSer129 $\alpha$-syn aggregates. The mechanisms underlying the accelerating role of G2019S LRRK2 on $\alpha$-syn toxicity need to be identified, and even if no changes in microglia were detected at 12 or 20 weeks after AAV2/9-ho-syn injection, whether G2019S LRRK2 accelerates synucleinopathy by modulating the microglial response needs to be investigated at earlier time-points. The present study therefore offers a model to study the interaction between LRRK2 G2019S, $\alpha$-syn and aging in the development of PD. G2019S KI mice show an increase of dopaminergic transmission at 1-3 months that normalizes at 12 months (Volta et al., 2017), i.e. by the time when striatal DAT levels and activity rise, striatal VMAT2 levels drop, and striatal and nigral pSer129 $\alpha$-syn levels increase (Longo et al., 2017). Early elevation of DA 
transmission and its later compensation via DAT elevation (stimulus-evoked DA release in unchanged in old mice) (Longo et al., 2017; Volta et al., 2017) might lead to enhanced cytosolic DA levels, somehow triggering pSer129 inclusions. In fact, DA can facilitate $\alpha$-syn aggregation through the formation of DA/ $\alpha$-syn complexes causing the blockade of chaperone-mediated autophagy (Martinez-Vicente et al., 2008) or of the process of $\alpha$-syn fibrillization (Outeiro et al., 2009). This is also relevant in vivo where elevated levels of DA can accelerate the formation of soluble $\alpha$-syn oligomers and hence DA cell death (Mor et al., 2017). In this respect, the present study suggests that pSer129 $\alpha$-syn signal in old G2019S KI mice is represented by PK-sensitive soluble forms of $\alpha$-syn. This confirms the view that the neuropathological process driven by G2019S LRRK2 is at the beginning, and that G2019S KI mice might represent a model of presymptomatic PD, characterized by early dysfunction at the dopaminergic synapse and defective $\alpha$-syn proteostasis (Longo et al., 2017).

Funding: this work has been supported by grants from the University of Ferrara and from the Italian Ministry of Health (\#RF-2011-02349806 to M.M). 


\section{Figure legends}

Figure 1. Behavioral impairment and nigrostriatal degeneration associated with ho-syn overexpression in 3-month-old mice. AAV2/9-ha-syn or AAV2/9 GFP (as a control) were injected bilaterally in the SNc of G2019S KI and WT mice. Stepping activity was evaluated using the drag test at baseline and 4, 8, 12, 16 and 20 weeks after surgery (A), stereological quantification of nigral dopamine neurons (B) and analysis of density of striatal tyrosine hydroxylase (TH) positive terminals (C) were performed after 20 weeks. Representative images of SNc and striatum are also given. Data are expressed as number of steps (A), number of nigral neurons (B) and optical density (grey scale arbitrary units, C) are means \pm SEM of 7 (G2019S KI GFP), 8 (LRRK2 A53T WT) and 9 (LRRK2 WT GFP and G2019S KI A53T) mice per group. Statistical analysis was performed by two-way RM ANOVA followed by the Bonferroni test for multiple comparisons (A) or one-way ANOVA followed by the Bonferroni test for multiple comparisons $(\mathrm{B}-\mathrm{C}) . * * \mathrm{P}<0.01$ different from respective GFP controls.

Figure 2. Expression of h $\alpha$-syn in 3-month-old mice. AAV2/9-h $\alpha$-syn or AAV2/9 GFP (as a control) were injected bilaterally in the SNc of G2019S KI and WT mice and transgene expression evaluated after 20 weeks. Representative images of SNc and striatum, and quantification of the area occupied by ha-syn immunostaining in the SNc (A) or striatum (B). Data are expressed as mean percentage \pm SEM of immunopositive surface of the structure of interest of 8 mice per group. Statistical analysis was performed by one-way ANOVA followed by the Bonferroni test for multiple comparisons. $* * \mathrm{P}<0.01$ different from respective GFP controls.

Figure 3. Increase of pSer129 $\alpha$-syn levels following ha-syn overexpression in 3-month-old mice. AAV2/9-ho-syn or AAV2/9 GFP (as a control) were injected bilaterally in the SNc of G2019S KI and WT mice, and pSer129 $\alpha$-syn or total $\alpha$-syn levels evaluated after 20 weeks. Representative 
images of total $\alpha$-syn signal in SNc and striatum, or pSer129 $\alpha$-syn signal in SNc and striatum, and relative quantifications (A, B, C, D, respectively). Data are expressed as mean percentage \pm SEM of immunopositive surface of the structure of interest of 8 mice per group. Statistical analysis was performed by one-way ANOVA followed by the Bonferroni test for multiple comparisons. $* * \mathrm{P}<0.01$ different from respective GFP controls

Figure 4. pSer129 $\alpha$-syn aggregates following ha-syn overexpression in 3-month-old mice. AAV2/9-ha-syn or AAV2/9 GFP (as a control) were injected bilaterally in the SNc of G2019S KI and WT mice and pSer129 $\alpha$-syn signal following Proteinase K (PK) digestion evaluated after 20 weeks. Representative images of SNc and striatum, and relative quantifications of pSer129 $\alpha$-syn signal (A, B, respectively). Data are expressed as mean percentage \pm SEM of immunopositive surface of the structure of interest of 8 mice per group. Statistical analysis was performed by oneway ANOVA followed by the Bonferroni test for multiple comparisons. $* * \mathrm{P}<0.01$ different from respective GFP controls

Figure 5. Behavioral impairment and nigrostriatal degeneration associated with ha-syn overexpression in 12-month-old mice. AAV2/9-h $\alpha$-syn or AAV2/9 GFP (as a control) were injected bilaterally in the SNc of G2019S KI and WT mice. Stepping activity was evaluated using the drag test (A) or the rotarod test (B) at baseline and 4, 8, 12 weeks after surgery, and nigrostriatal degeneration was evaluated after 12 weeks. Representative images of SNc and striatum are also given. Data are expressed as number of steps (A), time on rod (in sec; B), number of nigral neurons (C) and optical density (grey scale arbitrary units, B) are means \pm SEM of $n=7$ (LRRK2 WT GFP), 8 (LRRK2 WT A53T) and 10 (G2019S KI GFP and G2019S KI A53T) mice per group. Statistical analysis was performed by two-way RM ANOVA followed by the Bonferroni test for multiple comparisons (A-B) or one-way ANOVA followed by the Bonferroni test for multiple comparisons 
(C-D). ${ }^{* *} \mathrm{P}<0.01$ different from respective GFP controls, ${ }^{\circ} \mathrm{P}<0.05$ different from LRRK2 WT A53T.

Figure 6. Expression of ha-syn in 12-month-old mice. AAV2/9-h $\alpha$-syn or AAV2/9 GFP (as a control) were injected bilaterally in the SNc of G2019S KI and WT mice and transgene expression evaluated after 12 weeks. Representative images of SNc and striatum, and quantification of the area occupied by ha-syn immunostaining in the $\mathrm{SNc}(\mathrm{A})$ or striatum (B). Data are expressed as mean percentage \pm means \pm SEM of $n=7($ LRRK2 WT GFP), 8 (LRRK2 WT A53T) and $10($ G2019S KI GFP and G2019S KI A53T) mice per group. Statistical analysis was performed by one-way ANOVA followed by the Bonferroni test for multiple comparisons. ** $\mathrm{P}<0.01$ different from respective GFP controls.

Figure 7. Increase of pSer129 $\alpha$-syn levels following h $\alpha$-syn overexpression in 12-month-old mice. AAV2/9-ha-syn or AAV2/9 GFP (as a control) were injected bilaterally in the SNc of G2019S KI and WT mice, and pSer129 $\alpha$-syn or total $\alpha$-syn levels evaluated after 12 weeks. Representative images of total $\alpha$-syn signal in SNc and striatum, or pSer129 $\alpha$-syn signal in SNc and striatum, and relative quantifications (A, B, C, D, respectively), data are expressed as mean percentage \pm SEM of immunopositive surface of the structure of interest of 9 mice per group. Statistical analysis was performed by one-way ANOVA followed by the Bonferroni test for multiple comparisons. ** $\mathrm{P}<0.01$ different from respective GFP controls; ${ }^{\# \#} \mathrm{P}<0.01$ different from LRRK2 WT GFP; ${ }^{\circ} \mathrm{P}<0.01$ different from LRRK2 WT A53T.

Figure 8. pSer129 $\alpha$-syn aggregates following h $\alpha$-syn overexpression in 12-month-old mice. AAV2/9-ha-syn or AAV2/9 GFP (as a control) were injected bilaterally in the SNc of G2019S KI and WT mice, and pSer129 $\alpha$-syn signal following Proteinase K (PK) digestion evaluated after 12 
weeks. Representative images of SNc and striatum, and relative quantifications of pSer129 $\alpha$-syn signal (A, B, respectively). Data are expressed as mean percentage \pm SEM of immunopositive surface of the structure of interest of 9 mice per group. Statistical analysis was performed by oneway ANOVA followed by the Bonferroni test for multiple comparisons. $* * \mathrm{P}<0.01$ different from respective GFP controls; ${ }^{\circ} \mathrm{P}<0.01$ different from LRRK2 WT A53T. 


\section{References}

Anderson, J. P., et al., 2006. Phosphorylation of Ser-129 is the dominant pathological modification of alpha-synuclein in familial and sporadic Lewy body disease. J Biol Chem. 281, 29739-52.

Angot, E., et al., 2010. Are synucleinopathies prion-like disorders? Lancet Neurol. 9, 1128-38.

Arcuri, L., et al., 2016. Genetic and pharmacological evidence that endogenous nociceptin/orphanin FQ contributes to dopamine cell loss in Parkinson's disease. Neurobiol Dis. 89, 55-64.

Bengoa-Vergniory, N., et al., 2017. Alpha-synuclein oligomers: a new hope. Acta Neuropathol. $134,819-838$.

Bourdenx, M., et al., 2015. Lack of additive role of ageing in nigrostriatal neurodegeneration triggered by alpha-synuclein overexpression. Acta Neuropathol Commun. 3, 46.

Brundin, P., et al., 2017. Therapeutic approaches to target alpha-synuclein pathology. Exp Neurol. 298, 225-235.

Brundin, P., et al., 2010. Prion-like transmission of protein aggregates in neurodegenerative diseases. Nat Rev Mol Cell Biol. 11, 301-7.

Choi, I., et al., 2015. LRRK2 G2019S mutation attenuates microglial motility by inhibiting focal adhesion kinase. Nat Commun. 6, 8255.

Chu, Y., Kordower, J. H., 2007. Age-associated increases of alpha-synuclein in monkeys and humans are associated with nigrostriatal dopamine depletion: Is this the target for Parkinson's disease? Neurobiol Dis. 25, 134-49.

Chung, C. Y., et al., 2009. Dynamic changes in presynaptic and axonal transport proteins combined with striatal neuroinflammation precede dopaminergic neuronal loss in a rat model of AAV alphasynucleinopathy. J Neurosci. 29, 3365-73.

Cookson, M. R., 2010. The role of leucine-rich repeat kinase 2 (LRRK2) in Parkinson's disease. Nat Rev Neurosci. 11, 791-7. 
Correia Guedes, L., et al., 2010. Worldwide frequency of G2019S LRRK2 mutation in Parkinson's disease: a systematic review. Parkinsonism Relat Disord. 16, 237-42.

Cuervo, A. M., et al., 2004. Impaired degradation of mutant alpha-synuclein by chaperone-mediated autophagy. Science. 305, 1292-5.

Daher, J. P., et al., 2015. Leucine-rich Repeat Kinase 2 (LRRK2) Pharmacological Inhibition Abates alpha-Synuclein Gene-induced Neurodegeneration. J Biol Chem. 290, 19433-44.

Daher, J. P., et al., 2012. Neurodegenerative phenotypes in an A53T alpha-synuclein transgenic mouse model are independent of LRRK2. Hum Mol Genet. 21, 2420-31.

Daher, J. P., et al., 2014. Abrogation of alpha-synuclein-mediated dopaminergic neurodegeneration in LRRK2-deficient rats. Proc Natl Acad Sci U S A. 111, 9289-94.

Engeln, M., et al., 2013. Levodopa gains psychostimulant-like properties after nigral dopaminergic loss. Ann Neurol. 74, 140-4.

Fujiwara, H., et al., 2002. alpha-Synuclein is phosphorylated in synucleinopathy lesions. Nat Cell Biol. 4, 160-4.

Giasson, B. I., Lee, V. M., 2003. Are ubiquitination pathways central to Parkinson's disease? Cell. $114,1-8$.

Gillardon, F., et al., 2012. Parkinson's disease-linked leucine-rich repeat kinase 2(R1441G) mutation increases proinflammatory cytokine release from activated primary microglial cells and resultant neurotoxicity. Neuroscience. 208, 41-8.

Greggio, E., et al., 2011. Leucine-rich repeat kinase 2 and alpha-synuclein: intersecting pathways in the pathogenesis of Parkinson's disease? Mol Neurodegener. 6, 6.

Greggio, E., et al., 2006. Kinase activity is required for the toxic effects of mutant LRRK2/dardarin. Neurobiol Dis. 23, 329-41.

Guerreiro, P. S., et al., 2013. LRRK2 interactions with alpha-synuclein in Parkinson's disease brains and in cell models. J Mol Med (Berl). 91, 513-22. 
Herzig, M. C., et al., 2012. High LRRK2 levels fail to induce or exacerbate neuronal alphasynucleinopathy in mouse brain. PLoS One. 7, e36581.

Herzig, M. C., et al., 2011. LRRK2 protein levels are determined by kinase function and are crucial for kidney and lung homeostasis in mice. Hum Mol Genet. 20, 4209-23.

Ip, C. W., et al., 2017. AAV1/2-induced overexpression of A53T-alpha-synuclein in the substantia nigra results in degeneration of the nigrostriatal system with Lewy-like pathology and motor impairment: a new mouse model for Parkinson's disease. Acta Neuropathol Commun. 5, 11.

Kalia, L. V., et al., 2015. Clinical correlations with Lewy body pathology in LRRK2-related Parkinson disease. JAMA Neurol. 72, 100-5.

Kim, B., et al., 2012. Impaired inflammatory responses in murine Lrrk2-knockdown brain microglia. PLoS One. 7, e34693.

Kim, K. S., et al., 2018. Regulation of myeloid cell phagocytosis by LRRK2 via WAVE2 complex stabilization is altered in Parkinson's disease. Proc Natl Acad Sci U S A. 115, E5164-E5173.

Kritzinger, A., et al., 2018. Age-related pathology after adenoviral overexpression of the leucinerich repeat kinase 2 in the mouse striatum. Neurobiol Aging. 66, 97-111.

Larsen, J. O., et al., 1998. Global spatial sampling with isotropic virtual planes: estimators of length density and total length in thick, arbitrarily orientated sections. J Microsc. 191, 238-248.

Lashuel, H. A., et al., 2013. The many faces of alpha-synuclein: from structure and toxicity to therapeutic target. Nat Rev Neurosci. 14, 38-48.

Lauwers, E., et al., 2003. Neuropathology and neurodegeneration in rodent brain induced by lentiviral vector-mediated overexpression of alpha-synuclein. Brain Pathol. 13, 364-72.

Lee, H. J., et al., 2004. Clearance of alpha-synuclein oligomeric intermediates via the lysosomal degradation pathway. J Neurosci. 24, 1888-96.

Lin, X., et al., 2009. Leucine-rich repeat kinase 2 regulates the progression of neuropathology induced by Parkinson's-disease-related mutant alpha-synuclein. Neuron. 64, 807-27. 
Litteljohn, D., et al., 2018. The impact of murine LRRK2 G2019S transgene overexpression on acute responses to inflammatory challenge. Brain Behav Immun. 67, 246-256.

Longo, F., et al., 2017. Age-dependent dopamine transporter dysfunction and Serine129 phosphoalpha-synuclein overload in G2019S LRRK2 mice. Acta Neuropathol Commun. 5, 22.

Low, K., Aebischer, P., 2012. Use of viral vectors to create animal models for Parkinson's disease. Neurobiol Dis. 48, 189-201.

Marras, C., et al., 2011. Phenotype in parkinsonian and nonparkinsonian LRRK2 G2019S mutation carriers. Neurology. 77, 325-33.

Marti, M., et al., 2005. Blockade of nociceptin/orphanin FQ transmission attenuates symptoms and neurodegeneration associated with Parkinson's disease. J Neurosci. 25, 9591-601.

Marti, M., et al., 2004. Blockade of nociceptin/orphanin FQ receptor signaling in rat substantia nigra pars reticulata stimulates nigrostriatal dopaminergic transmission and motor behavior. $\mathbf{J}$ Neurosci. 24, 6659-66.

Martinez-Vicente, M., et al., 2008. Dopamine-modified alpha-synuclein blocks chaperone-mediated autophagy. J Clin Invest. 118, 777-88.

Mata, I. F., et al., 2006. LRRK2 in Parkinson's disease: protein domains and functional insights. Trends Neurosci. 29, 286-93.

Mattson, M. P., Magnus, T., 2006. Ageing and neuronal vulnerability. Nat Rev Neurosci. 7, 278-94.

Moehle, M. S., et al., 2015. The G2019S LRRK2 mutation increases myeloid cell chemotactic responses and enhances LRRK2 binding to actin-regulatory proteins. Hum Mol Genet. 24, 425067.

Moehle, M. S., et al., 2012. LRRK2 inhibition attenuates microglial inflammatory responses. J Neurosci. 32, 1602-11.

Mor, D. E., et al., 2017. Dopamine induces soluble alpha-synuclein oligomers and nigrostriatal degeneration. Nat Neurosci. 20, 1560-1568. 
Nalls, M. A., et al., 2014. Large-scale meta-analysis of genome-wide association data identifies six new risk loci for Parkinson's disease. Nat Genet. 46, 989-93.

Orenstein, S. J., et al., 2013. Interplay of LRRK2 with chaperone-mediated autophagy. Nat Neurosci. 16, 394-406.

Outeiro, T. F., et al., 2009. Dopamine-induced conformational changes in alpha-synuclein. PLoS One. 4, e6906.

Paisan-Ruiz, C., et al., 2004. Cloning of the gene containing mutations that cause PARK8-linked Parkinson's disease. Neuron. 44, 595-600.

Paxinos, G., Franklin, K. B. J., 2001. The mouse brain in stereotaxic coordinates. Academic Press, San Diego.

Poewe, W., et al., 2017. Parkinson disease. Nat Rev Dis Primers. 3, 17013.

Polymeropoulos, M. H., et al., 1997. Mutation in the alpha-synuclein gene identified in families with Parkinson's disease. Science. 276, 2045-7.

Rozas, G., Labandeira Garcia, J. L., 1997. Drug-free evaluation of rat models of parkinsonism and nigral grafts using a new automated rotarod test. Brain Res. 749, 188-99.

Russo, I., et al., 2015. Leucine-rich repeat kinase 2 positively regulates inflammation and downregulates NF-kappaB p50 signaling in cultured microglia cells. J Neuroinflammation. 12, 230.

Saha, S., et al., 2015. Mutations in LRRK2 potentiate age-related impairment of autophagic flux. Mol Neurodegener. 10, 26.

Sanchez-Guajardo, V., et al., 2010. Microglia acquire distinct activation profiles depending on the degree of alpha-synuclein neuropathology in a rAAV based model of Parkinson's disease. PLoS One. 5, e8784.

Satake, W., et al., 2009. Genome-wide association study identifies common variants at four loci as genetic risk factors for Parkinson's disease. Nat Genet. 41, 1303-7. 
Schallert, T., et al., 1979. Excessive bracing reactions and their control by atropine and L-DOPA in an animal analog of Parkinsonism. Exp Neurol. 64, 33-43.

Schapansky, J., et al., 2018. Familial knockin mutation of LRRK2 causes lysosomal dysfunction and accumulation of endogenous insoluble alpha-synuclein in neurons. Neurobiol Dis. 111, 26-35. Singleton, A. B., et al., 2003. alpha-Synuclein locus triplication causes Parkinson's disease. Science. $302,841$.

Song, L. K., et al., 2015. Targeted Overexpression of alpha-Synuclein by rAAV2/1 Vectors Induces Progressive Nigrostriatal Degeneration and Increases Vulnerability to MPTP in Mouse. PLoS One. 10, e0131281.

Spillantini, M. G., et al., 1997. Alpha-synuclein in Lewy bodies. Nature. 388, 839-40.

Stefanis, L., 2012. alpha-Synuclein in Parkinson's disease. Cold Spring Harb Perspect Med. 2, a009399.

Svarcbahs, R., et al., 2016. Inhibition of Prolyl Oligopeptidase Restores Spontaneous Motor Behavior in the alpha-Synuclein Virus Vector-Based Parkinson's Disease Mouse Model by Decreasing alpha-Synuclein Oligomeric Species in Mouse Brain. J Neurosci. 36, 12485-12497.

Thome, A. D., et al., 2015. Fractalkine Signaling Regulates the Inflammatory Response in an alphaSynuclein Model of Parkinson Disease. PLoS One. 10, e0140566.

Ulusoy, A., et al., 2010. Viral vector-mediated overexpression of alpha-synuclein as a progressive model of Parkinson's disease. Prog Brain Res. 184, 89-111.

Van der Perren, A., et al., 2015. Viral vector-based models of Parkinson's disease. Curr Top Behav Neurosci. 22, 271-301.

Viaro, R., et al., 2008. Nociceptin/orphanin FQ receptor blockade attenuates MPTP-induced parkinsonism. Neurobiol Dis. 30, 430-8.

Volpicelli-Daley, L. A., et al., 2016. G2019S-LRRK2 Expression Augments alpha-Synuclein Sequestration into Inclusions in Neurons. J Neurosci. 36, 7415-27. 
Volta, M., et al., 2017. Initial elevations in glutamate and dopamine neurotransmission decline with age, as does exploratory behavior, in LRRK2 G2019S knock-in mice. Elife. 6.

West, A. B., et al., 2014. Differential LRRK2 expression in the cortex, striatum, and substantia nigra in transgenic and nontransgenic rodents. J Comp Neurol. 522, 2465-80.

West, A. B., et al., 2005. Parkinson's disease-associated mutations in leucine-rich repeat kinase 2 augment kinase activity. Proc Natl Acad Sci U S A. 102, 16842-7.

Xilouri, M., Stefanis, L., 2016. Chaperone mediated autophagy in aging: Starve to prosper. Ageing Res Rev. 32, 13-21.

Yao, C., et al., 2010. LRRK2-mediated neurodegeneration and dysfunction of dopaminergic neurons in a Caenorhabditis elegans model of Parkinson's disease. Neurobiol Dis. 40, 73-81.

Zimprich, A., et al., 2004. Mutations in LRRK2 cause autosomal-dominant parkinsonism with pleomorphic pathology. Neuron. 44, 601-7.

Zolotukhin, S., et al., 1999. Recombinant adeno-associated virus purification using novel methods improves infectious titer and yield. Gene Ther. 6, 973-85. 


\section{Figure 1}

Click here to download high resolution image
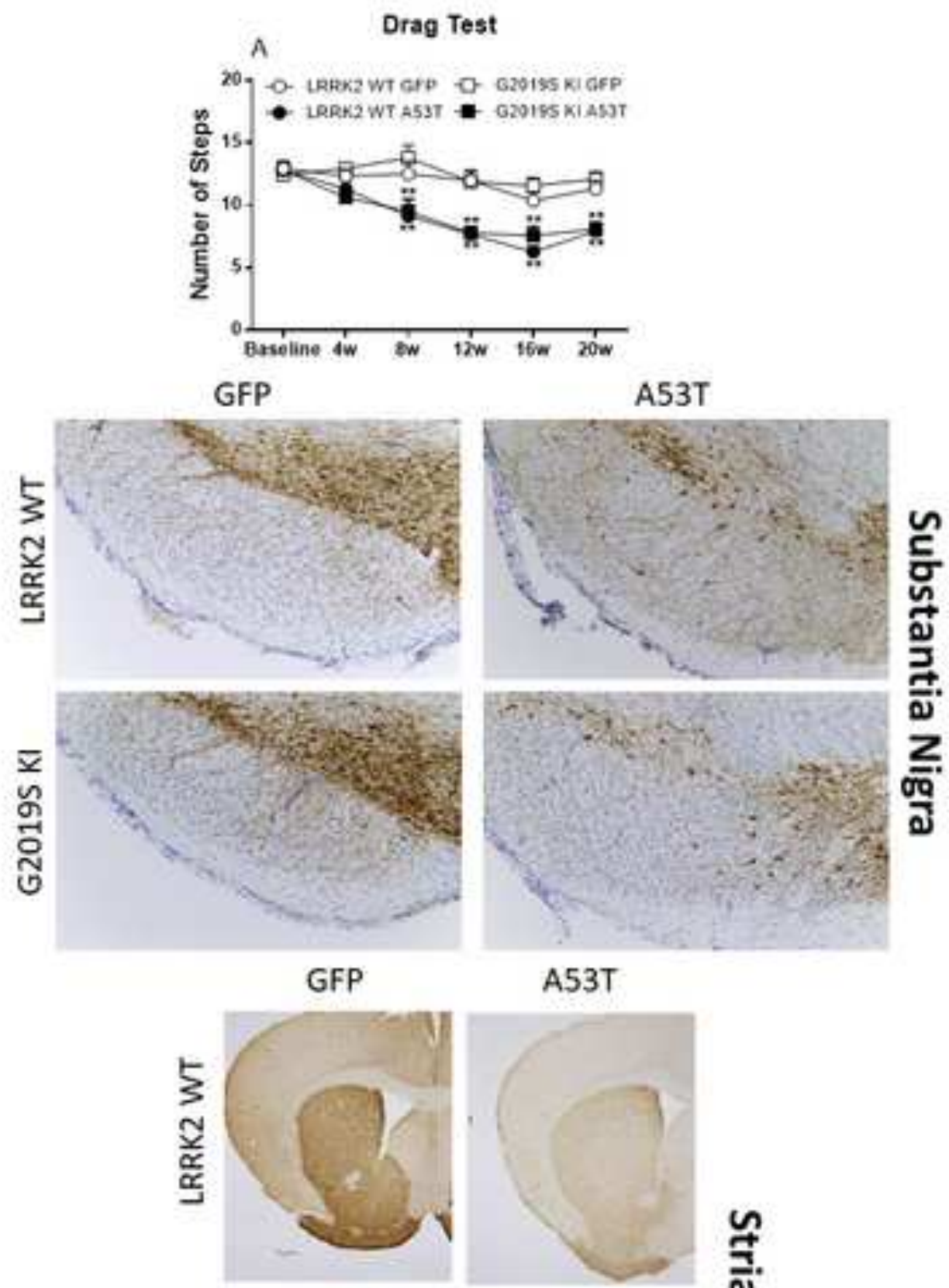

A53T
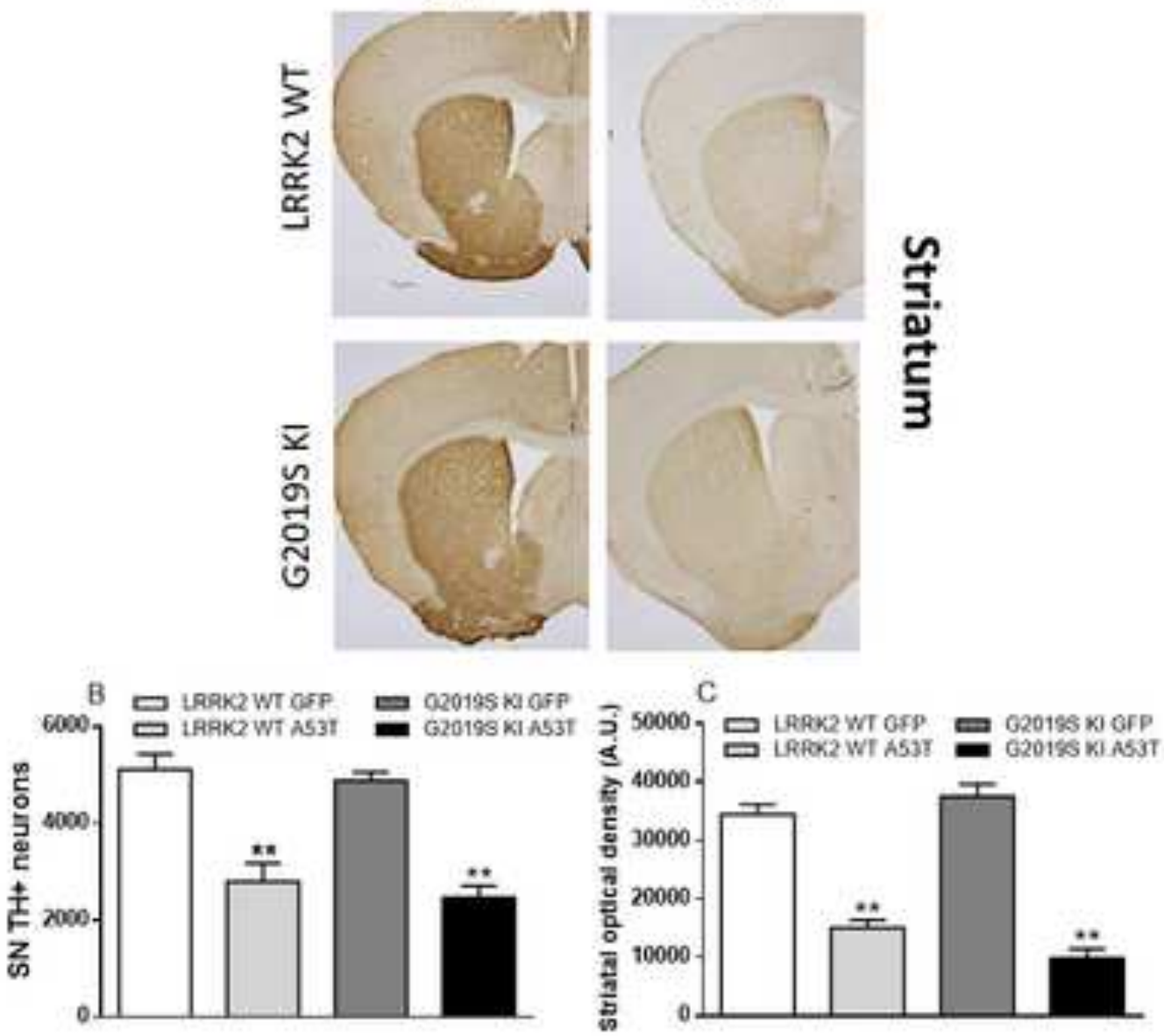
Figure 2
Click here to download high resolution image
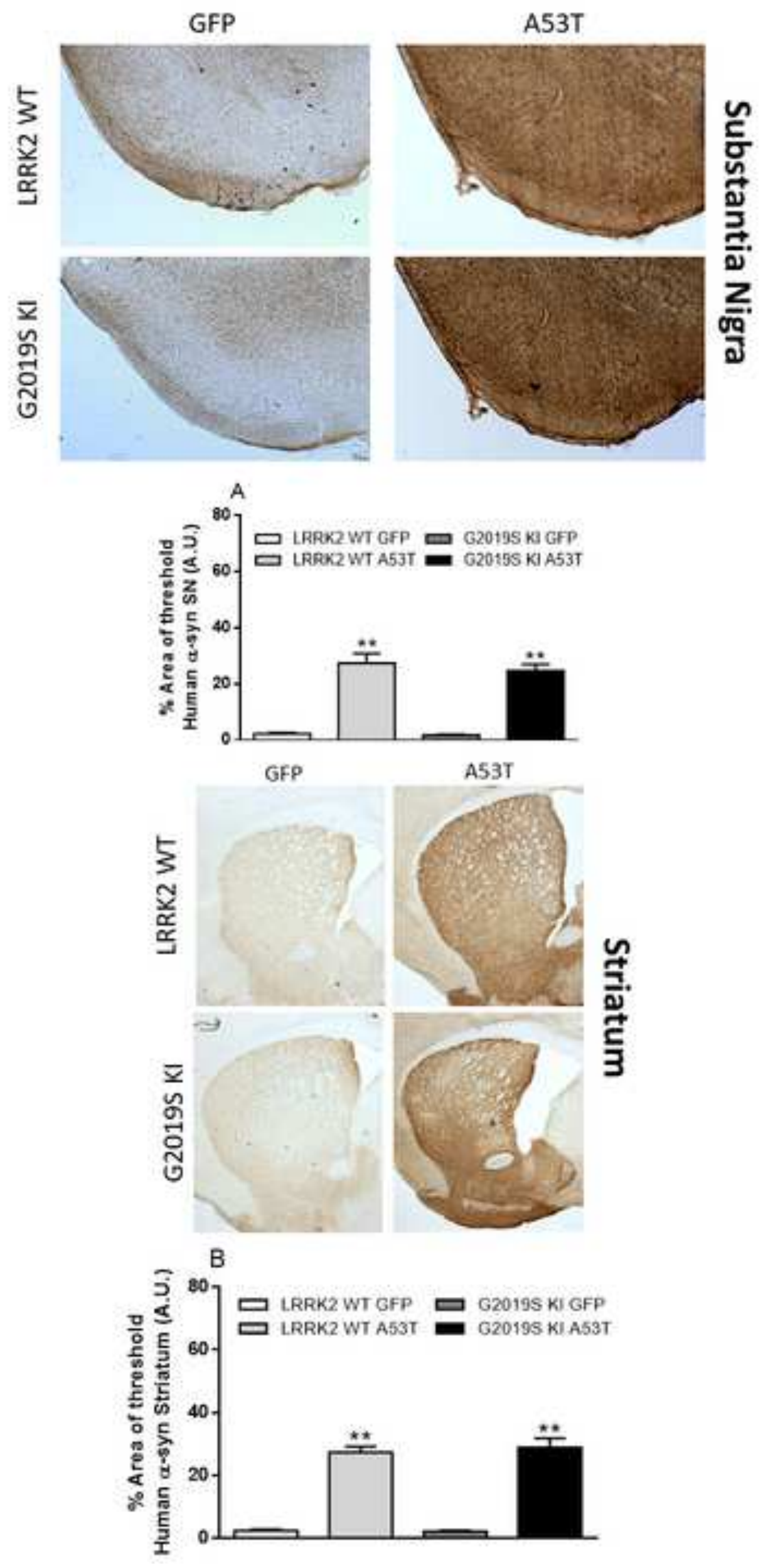


\section{Figure 3}

Click here to download high resolution image
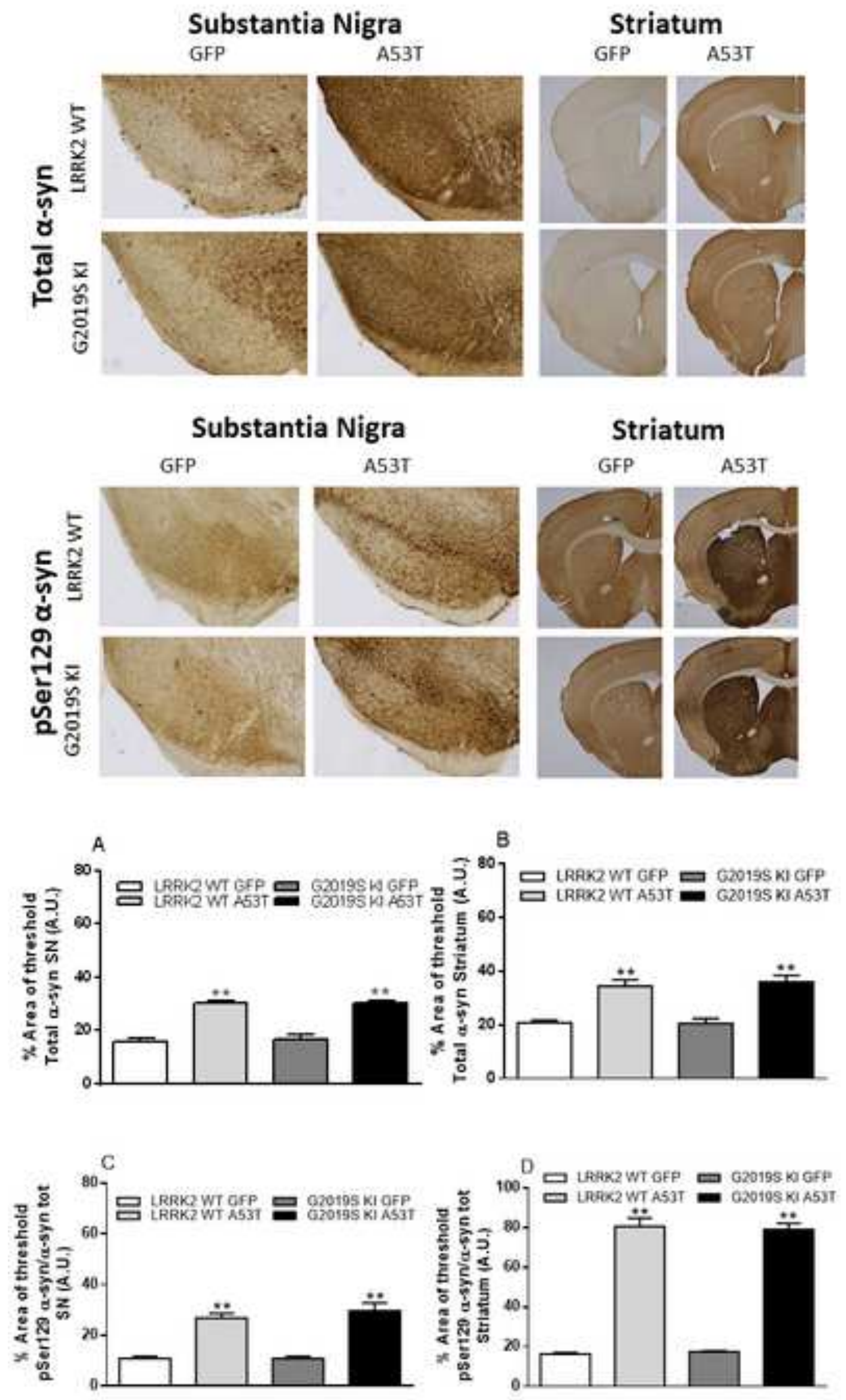

\section{Striatum}

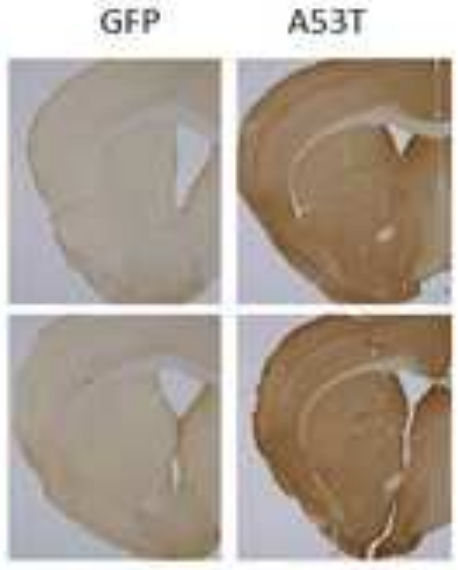

Striatum

GFP 
Figure 4

Click here to download high resolution image
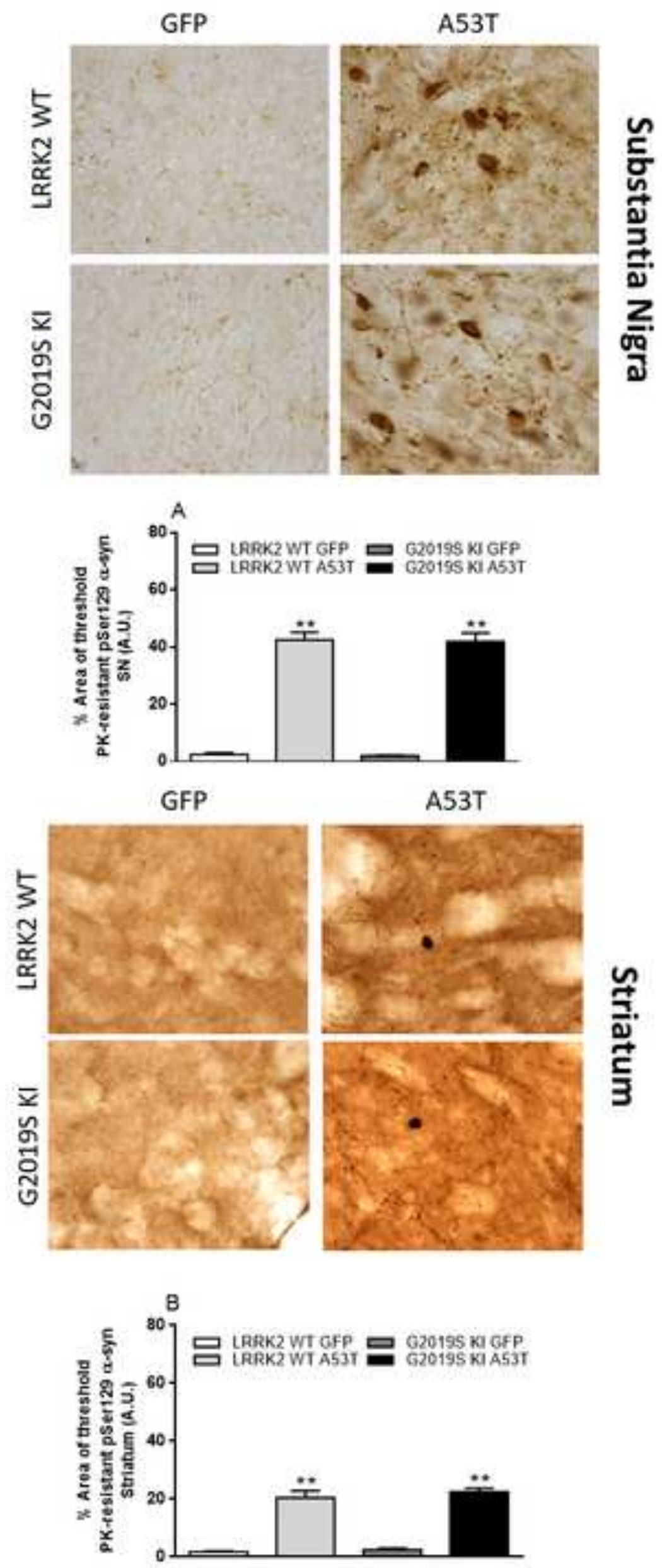
Figure 5

Click here to download high resolution image
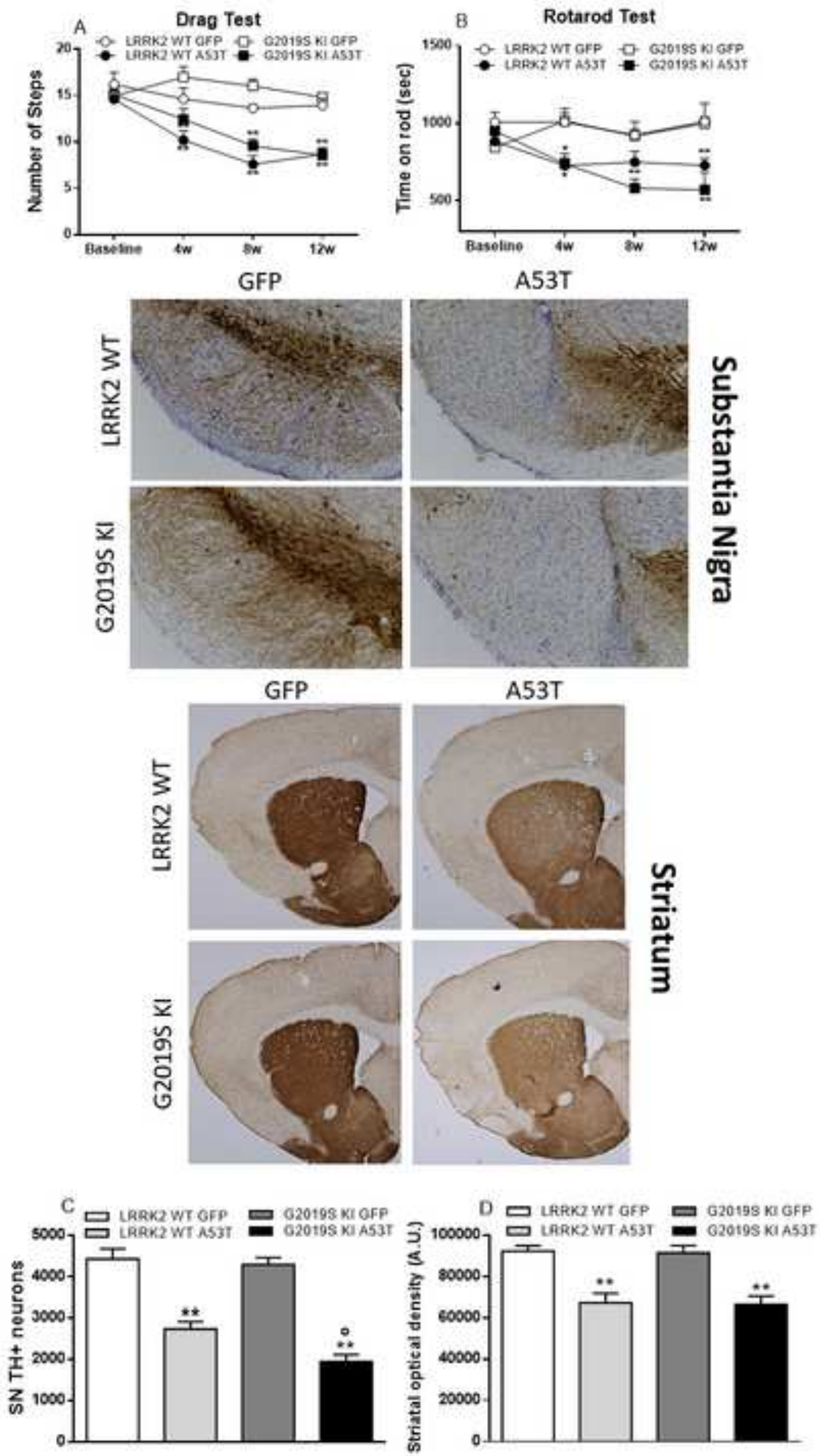
Figure 6
Click here to download high resolution image
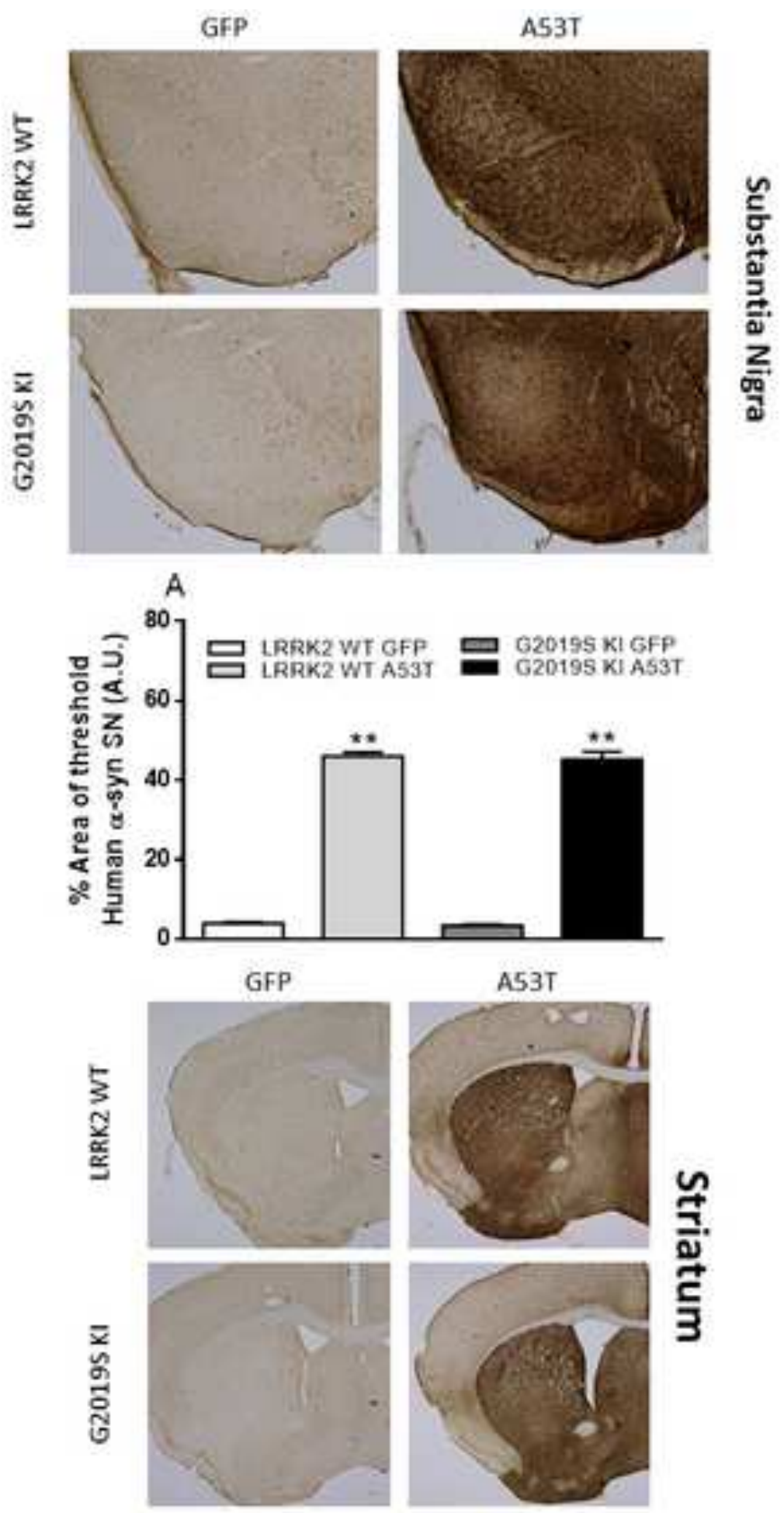

B

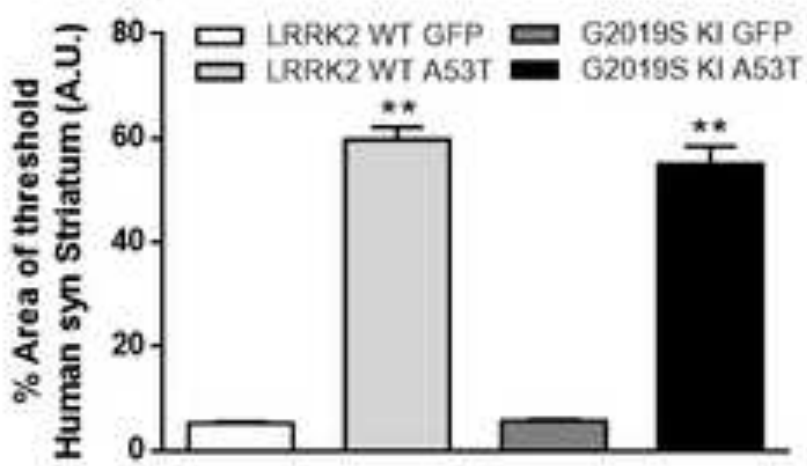




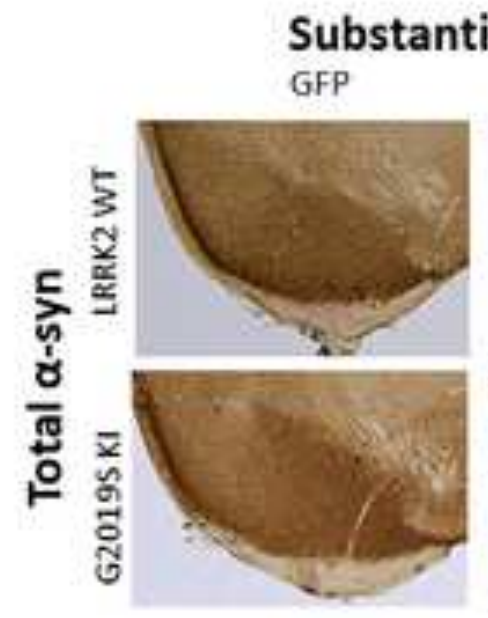

\section{Substantia Nigra}

A53T

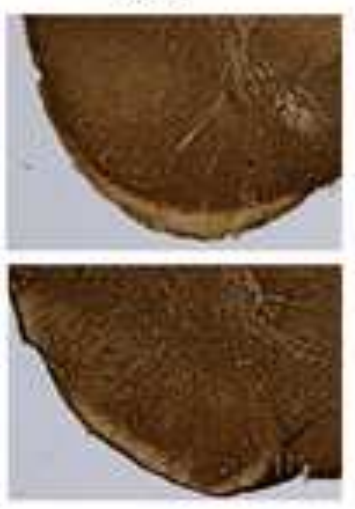

\section{Substantia Nigra} GFP

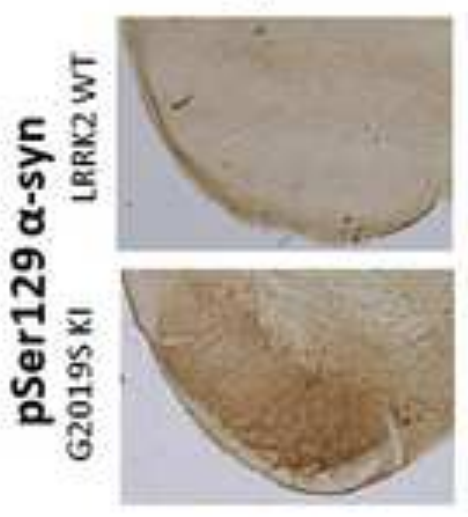

A53T

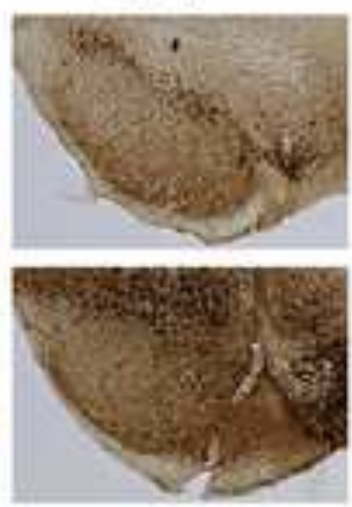

Striatum

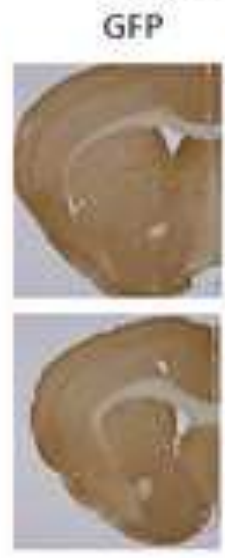

A53T

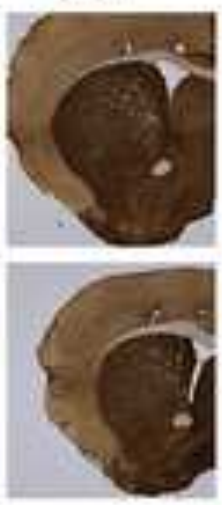

Striatum

GFP

A53T

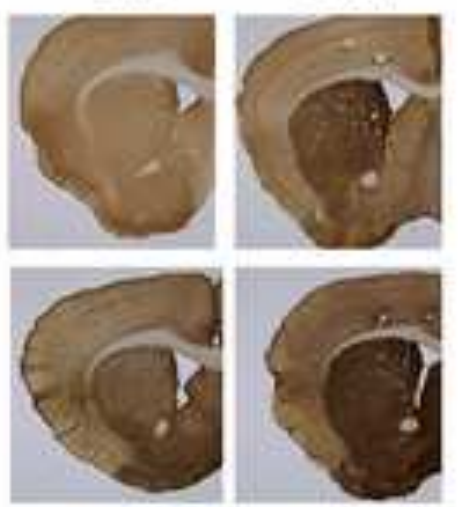

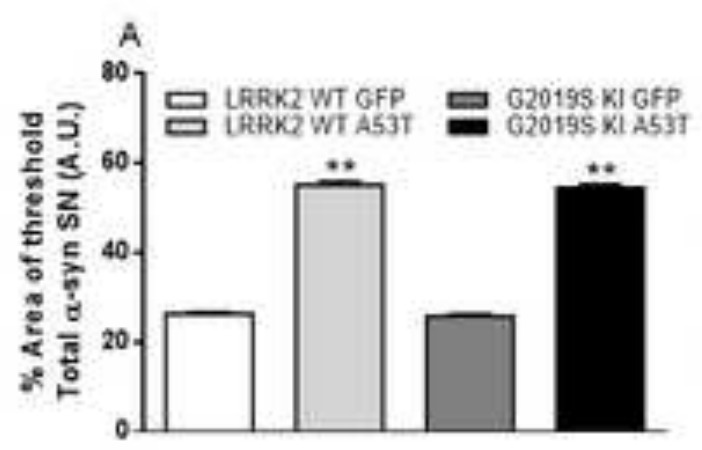
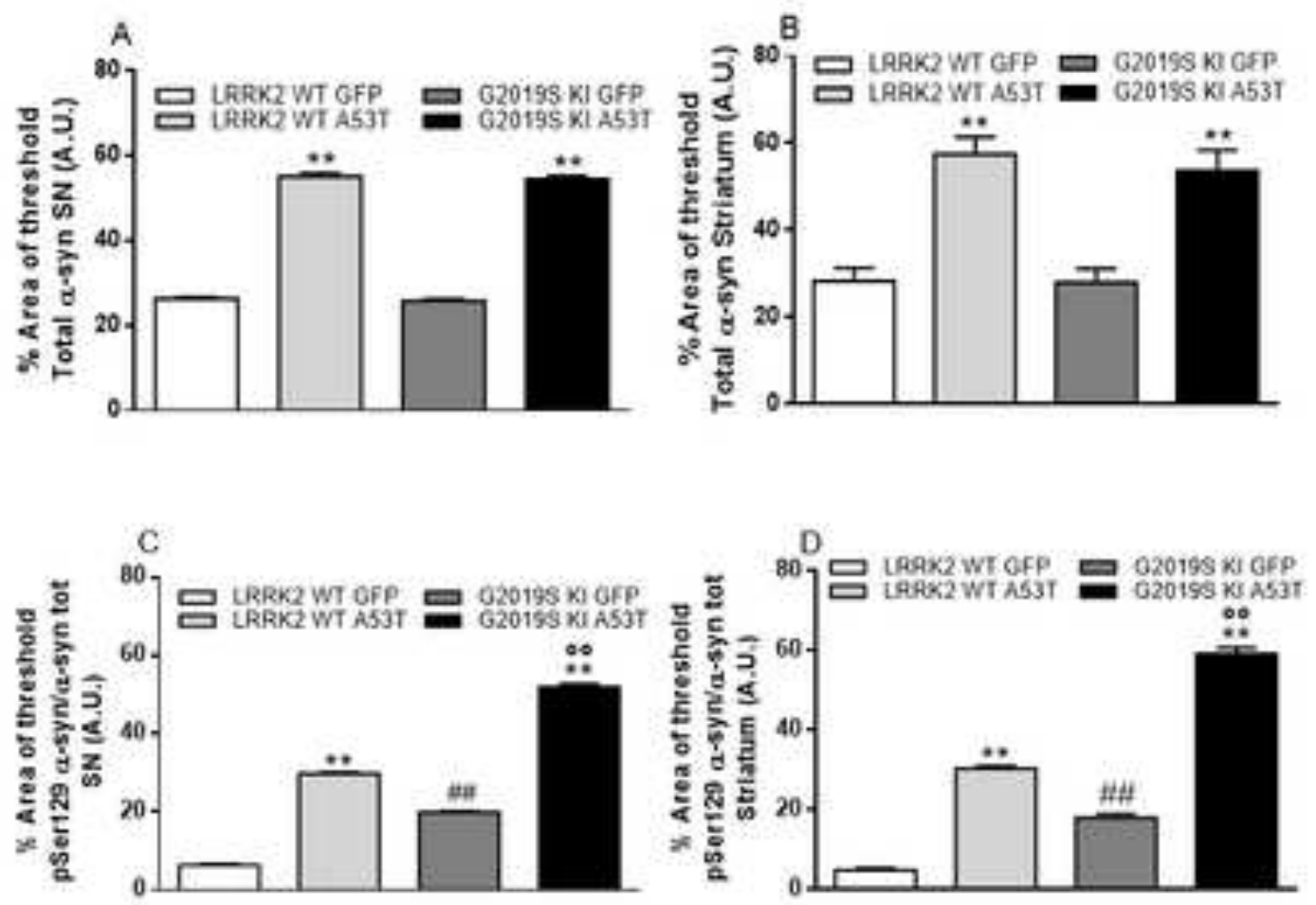
Click here to download high resolution image
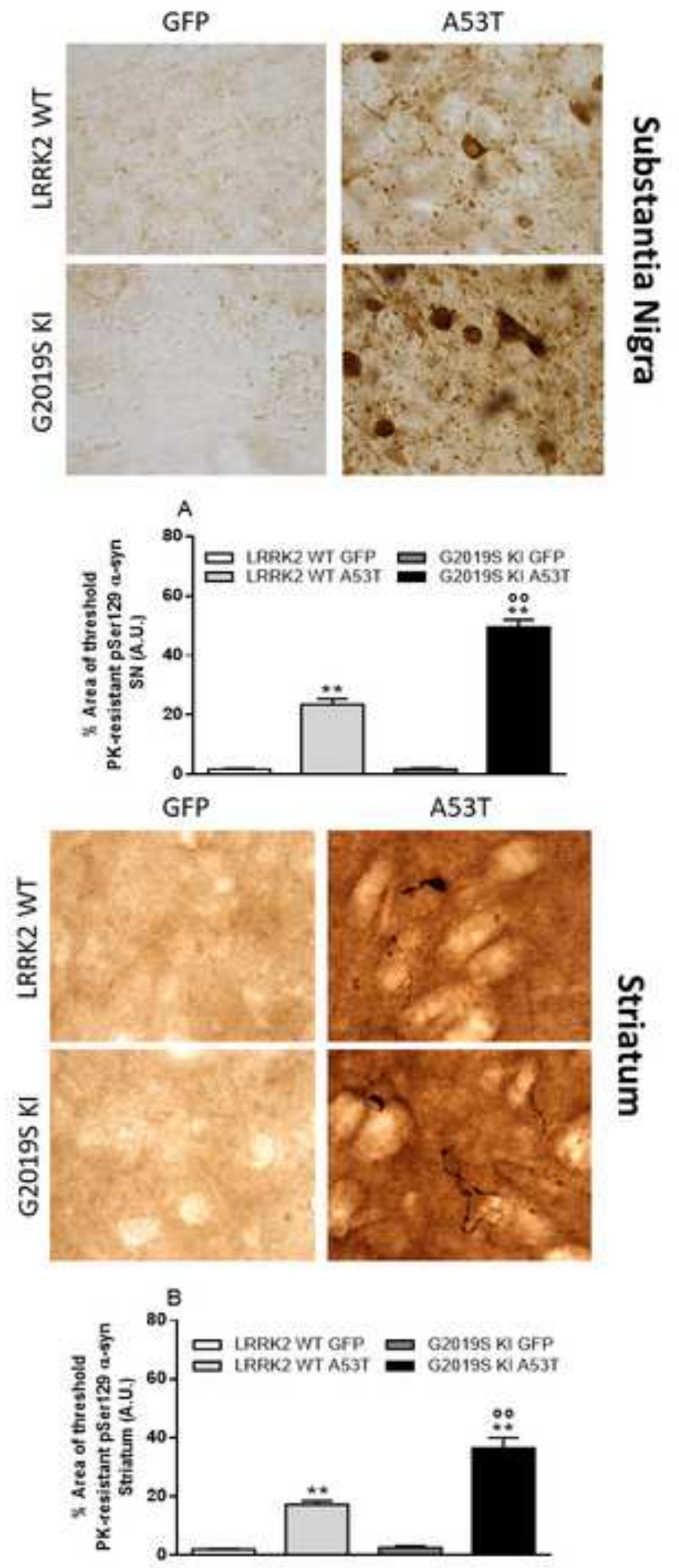
Supplementary Material
Click here to download Supplementary Material: Novello_NBD_Supplementary.docx

Supplementary Material
Click here to download Supplementary Material: Novello_NBD_Supplementary.docx

(a) (a) .

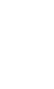

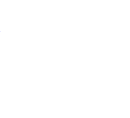

